\title{
QUADRATURE FORMULAS BASED ON RATIONAL INTERPOLATION
}

\author{
WALTER VAN ASSCHE AND INGRID VANHERWEGEN
}

\begin{abstract}
We consider quadrature formulas based on interpolation using the basis functions $1 /\left(1+t_{k} x\right) \quad(k=1,2,3, \ldots)$ on $[-1,1]$, where $t_{k}$ are parameters on the interval $(-1,1)$. We investigate two types of quadratures: quadrature formulas of maximum accuracy which correctly integrate as many basis functions as possible (Gaussian quadrature), and quadrature formulas whose nodes are the zeros of the orthogonal functions obtained by orthogonalizing the system of basis functions (orthogonal quadrature). We show that both approaches involve orthogonal polynomials with modified weights which depend on the number of quadrature nodes. The asymptotic distribution of the nodes is obtained as well as various interlacing properties and monotonicity results for the nodes.
\end{abstract}

\section{INTRODUCTION}

Suppose that we want to compute the integral

$$
\int_{-1}^{1} f(x) w(x) d x
$$

with $w(x)$ a positive and integrable weight function on $[-1,1]$; then one can use an interpolatory quadrature formula by approximating $f$ using interpolation. In this paper we assume that $w(x)>0$ almost everywhere in $[-1,1]$ and use interpolation by means of the basis functions

$$
\frac{1}{1+t_{1} x}, \frac{1}{1+t_{2} x}, \ldots, \frac{1}{1+t_{n} x}, \ldots,
$$

where $t_{1}, t_{2}, t_{3}, \ldots$ are parameters belonging to $(-1,1)$. We consider two approaches for choosing the interpolation nodes (quadrature nodes). The first consists of choosing the $n$ nodes to be the zeros of the orthogonal function $r_{n+1}$ obtained by orthogonalizing the system (1.1) using the inner product

$$
(f, g)=\int_{-1}^{1} f(x) g(x) w(x) d x .
$$

We refer to this as orthogonal quadrature. The second approach that we investigate is to choose the nodes in such a way that the quadrature formula is

Received by the editor March 27, 1992 and, in revised form, November 5, 1992.

1991 Mathematics Subject Classification. Primary 65D32, 41A20, 42C05.

Key words and phrases. Quadrature, rational interpolation, orthogonal polynomials.

The first author is a Research Associate of the Belgian National Fund for Scientific Research. 
correct for as many basis functions as possible. This gives a quadrature with maximum accuracy, and we refer to it as Gaussian quadrature. It is well known that both approaches are the same when one is using interpolation by polynomials, but these choices result in different quadrature formulas when one uses interpolation with the basis functions (1.1).

1.1. Orthogonal functions. It is well known that the zeros of the $n$ th-degree orthogonal polynomial with respect to a positive weight function $w(x)$ on $[-1,1]$ are all real, simple, and in the interval $(-1,1)$, and that they separate the zeros of the orthogonal polynomial of degree $n+1$ [26, pp. 44-46]. These properties can be generalized to other orthogonal systems of functions $\left\{\phi_{i}: i=1,2, \ldots\right\}$, where orthogonality is with respect to the weight function $w(x)$ on $[-1,1]$, provided they satisfy the Haar condition. The sequence of functions $\left\{\phi_{i}: i=1, \ldots, n\right\}$ satisfies the Haar condition on $[-1,1]$ if and only if for every choice of $n$ points $x_{i} \in[-1,1](i=1, \ldots, n)$ with $x_{i} \neq x_{j}$ whenever $i \neq j$ one has $\operatorname{det}_{1 \leq i, j \leq n}\left(\phi_{i}\left(x_{j}\right)\right) \neq 0$ (see, e.g., [14]). This condition is equivalent to saying that each linear combination $\phi=\sum_{i=1}^{n} a_{i} \phi_{i}$ has at most $n-1$ zeros in $[-1,1]$. A system $\left\{\phi_{i}: i=1, \ldots, n\right\}$ is an extended Haar system on $[-1,1]$ if and only if each linear combination $\phi=\sum_{i=1}^{n} a_{i} \phi_{i}$ has at most $n-1$ zeros in [-1,1], counting multiplicities. Pinkus and Ziegler [25] and Videnskii [28] have investigated several properties of systems of orthogonal functions $\left\{\phi_{i}: i=1,2, \ldots, n+1\right\}$ satisfying the Haar condition on $[-1,1]$. They have shown that $\phi_{n+1}$ has precisely $n$ zeros in $(-1,1)$, the multiplicity of each zero is odd, and the zeros of $\phi_{n}$ and $\phi_{n+1}$ are strictly interlacing.

Suppose that $t_{i} \in(-1,1) \quad(i=1,2, \ldots)$ and that $t_{i} \neq t_{j}$ whenever $i \neq j$. Then the system $\left\{\frac{1}{1+t_{1} x}, \frac{1}{1+t_{2} x}, \ldots, \frac{1}{1+t_{n+1} x}\right\}$ is an extended Haar system on $[-1,1]$ because

$$
\phi(x)=\sum_{i=1}^{n+1} a_{i} \phi_{i}(x)=\frac{p_{n}(x)}{\prod_{i=1}^{n+1}\left(1+t_{i} x\right)},
$$

where $p_{n}(x)$ is a polynomial of degree at most $n$, and hence $\phi$ has at most $n$ zeros on $[-1,1]$, counting multiplicities. If we apply the Gram-Schmidt procedure to $\left\{\frac{1}{1+t_{1} x}, \frac{1}{1+t_{2} x}, \ldots\right\}$, then we obtain a sequence of orthogonal functions on $[-1,1]$ with respect to the weight function $w(x)$. We denote these orthogonal functions by

$$
r_{n+1}\left(t_{1}, \ldots, t_{n+1} ; x\right)=\frac{1}{1+t_{n+1} x}+\sum_{i=1}^{n} \frac{c_{i}}{1+t_{i} x},
$$

and, as mentioned before, this rational function has precisely $n$ zeros in $(-1,1)$. It is well known that

$$
\min _{\left(a_{1}, \ldots, a_{n}\right) \in \mathbf{R}^{n}} \int_{-1}^{1}\left|\frac{1}{1+t_{n+1} x}-\sum_{i=1}^{n} \frac{a_{i}}{1+t_{i} x}\right|^{2} w(x) d x
$$

is obtained when

$$
\frac{1}{1+t_{n+1} x}-\sum_{i=1}^{n} \frac{a_{i}}{1+t_{i} x}=r_{n+1}\left(t_{1}, \ldots, t_{n} ; x\right),
$$

so that $r_{n+1}\left(t_{1}, \ldots, t_{n} ; x\right)$ is the error function for the best $L_{2}(w)$-approximation of $\frac{1}{1+t_{n+1} x}$ by linear combinations of $\left\{\frac{1}{1+t_{1} x}, \frac{1}{1+t_{2} x}, \ldots, \frac{1}{1+t_{n} x}\right\}$. 
1.2. Gaussian quadrature. Suppose the system of functions $\left\{\phi_{i}: i=1,2, \ldots\right\}$ is an extended Markov system on $[-1,1]$, i.e., $\left\{\phi_{i}: i=1,2, \ldots, k\right\}$ is an extended Haar system on $[-1,1]$ for each $k \in \mathbb{N}$. If one considers a quadrature formula with $n$ nodes which is of maximum accuracy, then one wants to integrate the functions $\phi_{i} \quad(1 \leq i \leq m)$ correctly for $m$ as large as possible. If $\phi_{i}(x)=x^{i-1}$, then $m$ turns out to be $2 n$, and the nodes are the zeros of the orthogonal polynomial of degree $n$ for the weight function $w(x)$ (Gauss-Jacobi quadrature). Krein [15] showed that when $\left\{\phi_{i}: i=1,2, \ldots, 2 n\right\}$ is a Haar system on $[-1,1]$, then there exists a unique set of $n$ nodes $x_{i} \in(-1,1)$ and $n$ strictly positive weights $\beta_{i}$ such that for $k=1,2, \ldots, 2 n$

$$
\int_{-1}^{1} \phi_{k}(x) w(x) d x=\sum_{i=1}^{n} \beta_{i} \phi_{k}\left(x_{i}\right)
$$

and this gives maximum accuracy. Karlin and Pinkus [12] have generalized this by allowing nodes with multiplicities, and there are further generalizations by Barrow [2] and Bojanov, Braess, and Dyn [3].

The system of rational functions (1.1) is an extended Markov system on $[-1,1]$, hence the properties mentioned above are all valid. In particular, it follows that the nodes for Gaussian quadrature are all in $(-1,1)$ and the corresponding quadrature weights are all strictly positive.

\section{CONNECTION WITH ORTHOGONAL POLYNOMIALS}

In this section we will make the simple observation that the nodes and weights for both orthogonal quadrature and Gaussian quadrature based on the rational functions (1.1) are closely related to the zeros and Christoffel numbers for orthogonal polynomials on $[-1,1]$ with a weight function that depends on the number of nodes. This observation is not new (see, e.g., López and Illán $[18,19]$ for Gaussian quadrature), but this interaction makes it possible to use results from the theory of orthogonal polynomials to obtain useful properties of the nodes and weights for quadrature based on rational interpolation.

Theorem 1. Let $\left\{\phi_{k}\right\}$ be the set of basis functions (1.1).

(1) Suppose all the $t_{i}$ are different; then the $n$ nodes for orthogonal quadrature are the zeros $x_{j, n}(1 \leq j \leq n)$ of the orthogonal polynomial $p_{n}(x)$ with respect to the weight function

$$
w_{n}(x)=\frac{w(x)}{\pi_{n}(x) \pi_{n+1}(x)}, \quad-1<x<1,
$$

where

$$
\pi_{n}(x)=\prod_{i=1}^{n}\left(1+t_{i} x\right) .
$$

If $t_{1}=0$, then (1.2) holds for $k=1,2, \ldots, n+1$ with $x_{j}=x_{j, n}$ and

$$
\beta_{j}\left(t_{1}, \ldots, t_{n+1}\right)=\pi_{n+1}\left(x_{j, n}\right) \pi_{n}\left(x_{j, n}\right) \lambda_{j, n},
$$

where the $\lambda_{j, n}$ are the Christoffel numbers corresponding to the orthogonal polynomial $p_{n}(x)$ for the weight function (2.1). 
(2) Suppose all the $t_{i}$ are different; then the nodes for Gaussian quadrature are the zeros $x_{j, n}(1 \leq j \leq n)$ of the orthogonal polynomial $p_{n}(x)$ with respect to the weight function

$$
w_{n}(x)=\frac{w(x)}{\pi_{2 n}(x)}, \quad-1<x<1,
$$

and (1.2) holds for $k=1,2, \ldots, 2 n$ with $x_{j}=x_{j, n}$ and

$$
\beta_{j}\left(t_{1}, \ldots, t_{2 n}\right)=\pi_{2 n}\left(x_{j, n}\right) \lambda_{j, n},
$$

where the $\lambda_{j, n}$ are the Christoffel numbers corresponding to the orthogonal polynomial $p_{n}(x)$ for the weight function (2.3).

Proof. (1) The orthogonal functions obtained by orthogonalizing the system of basis functions $(1.1)$ on $[-1,1]$ with weight function $w(x)$ are of the form

$$
r_{n+1}\left(t_{1}, \ldots, t_{n+1} ; x\right)=\sum_{k=1}^{n+1} \frac{a_{k}}{1+t_{k} x}=\frac{p_{n}(x)}{\pi_{n+1}(x)} \quad\left(a_{n+1}=1\right),
$$

where $p_{n}(x)$ is a polynomial of degree $n$. The orthogonality implies that

$$
\int_{-1}^{1} \frac{p_{n}(x)}{\pi_{n+1}(x)} \sum_{j=1}^{n} \frac{c_{j}}{1+t_{j} x} w(x) d x=0
$$

for every choice of parameters $c_{1}, c_{2}, \ldots, c_{n}$. This is the same as saying that

$$
\int_{-1}^{1} p_{n}(x) q_{n-1}(x) \frac{w(x)}{\pi_{n}(x) \pi_{n+1}(x)} d x=0
$$

for every polynomial $q_{n-1}(x)$ of degree at most $n-1$. This shows that $p_{n}(x)$ is the orthogonal polynomial of degree $n$ for the weight function (2.1). The Gauss-Jacobi quadrature for the weight function (2.1) gives

$$
\int_{-1}^{1} \frac{q_{2 n-1}(x) w(x)}{\pi_{n}(x) \pi_{n+1}(x)} d x=\sum_{j=1}^{n} \lambda_{j, n} q_{2 n-1}\left(x_{j, n}\right),
$$

where the $x_{j, n}$ are the zeros of $p_{n}(x)$ and $q_{2 n-1}(x)$ is a polynomial of degree at most $2 n-1$. If $t_{1}=0$ then $\pi_{n}(x)$ is a polynomial of degree $n-1$. In this case choose $q_{2 n-1}(x)=\pi_{n}(x) \pi_{n+1}(x) /\left(1+t_{k} x\right)$ to obtain

$$
\int_{-1}^{1} \frac{w(x)}{1+t_{k} x} d x=\sum_{j=1}^{n} \frac{\lambda_{j, n} \pi_{n}\left(x_{j, n}\right) \pi_{n+1}\left(x_{j, n}\right)}{1+t_{k} x_{j, n}}, \quad k=1,2, \ldots, n+1,
$$

from which the assertion follows.

(2) Let $x_{i} \equiv x_{i}\left(t_{1}, \ldots, t_{2 n}\right)$ and $\beta_{i} \equiv \beta_{i}\left(t_{1}, \ldots, t_{2 n}\right) \quad(i=1,2, \ldots, n)$. In order to obtain the nodes $x_{i}$ and weights $\beta_{i}$, one needs to solve the system of equations

$$
\int_{-1}^{1} \frac{w(x)}{1+t_{j} x} d x=\sum_{i=1}^{n} \frac{\beta_{i}}{1+t_{j} x_{i}}, \quad j=1,2, \ldots, 2 n .
$$

This is equivalent to finding $x_{i}$ and $\beta_{i}(i=1,2, \ldots, n)$ such that

$$
\int_{-1}^{1} \frac{q_{2 n-1}(x)}{\pi_{2 n}(x)} w(x) d x=\sum_{i=1}^{n} \beta_{i} \frac{q_{2 n-1}\left(x_{i}\right)}{\pi_{2 n}\left(x_{i}\right)}
$$


for every polynomial $q_{2 n-1}$ of degree at most $2 n-1$. From the theory of orthogonal polynomials (Gauss-Jacobi quadrature) we then know that the nodes $x_{i}$ are the zeros of the orthogonal polynomial of degree $n$ for the weight function (2.3) and then

$$
\int_{-1}^{1} \frac{q_{2 n-1}(x)}{\pi_{2 n}(x)} w(x) d x=\sum_{i=1}^{n} \lambda_{i, n} q_{2 n-1}\left(x_{i}\right) .
$$

Choose $q_{2 n-1}(x)=\pi_{2 n}(x) /\left(1+t_{k} x\right) \quad(k=1, \ldots, 2 n)$ to obtain the assertion.

In case the weight function $w(x)$ is equal to either

$$
w(x)=\left\{\begin{array}{l}
\frac{1}{\sqrt{1-x^{2}}}, \\
\sqrt{1-x^{2}}, \\
\sqrt{\frac{1-x}{1+x}},
\end{array}\right.
$$

the orthogonal polynomials for the modified weight functions $w_{n}(x)$ given by (2.1) or (2.3) can be found explicitly and are known as Bernstein-Szegö polynomials $[26$, p. 31$]$.

The nodes of both orthogonal quadrature and Gaussian quadrature are equal to the zeros of the orthogonal polynomial $p_{n}(x)$ with respect to a weight function depending on $n$. Therefore, the distribution of the quadrature nodes for orthogonal quadrature and Gaussian quadrature is given by the distribution of the zeros of orthogonal polynomials with a weight function depending on the degree $n$. The asymptotic zero distribution and various asymptotic results for such orthogonal polynomials have been investigated recently by Mhaskar and Saff [22-24], Gonchar, López, and Rakhmanov [11, 21] and López [16, 17, 20]. An almost immediate corollary to their results is:

Theorem 2. Suppose that the asymptotic distribution of the parameters $\left\{t_{i}: i \leq\right.$ $n\}$ is given by a measure $\nu$ on $[-1,1]$, i.e., for every continuous function $f$ on $[-1,1]$ we have

$$
\lim _{n \rightarrow \infty} \frac{1}{n} \sum_{j=1}^{n} f\left(t_{j}\right)=\int_{-1}^{1} f(x) d \nu(x) .
$$

If $\nu=p \delta_{-1}+q \delta_{1}+r \delta_{0}+[1-(p+q+r)] \nu_{0}$, where $\delta_{a}$ is the unit measure with all its mass concentrated at $a$, and $p, q, r>0, p+q+r \leq 1$, and

$$
\int_{-1}^{1} \log |t| d \nu_{0}(t)<\infty
$$

then the asymptotic distribution of the nodes for both orthogonal and Gaussian quadrature is given by the measure

$$
\mu=p \delta_{1}+q \delta_{-1}+r \mu_{0}+[1-(p+q+r)] \mu_{b},
$$

where $\mu_{0}$ is the arcsin measure on $[-1,1]$ with weight function

$$
\mu_{0}^{\prime}(x)=\frac{1}{\pi} \frac{1}{\sqrt{1-x^{2}}}
$$


and $\mu_{b}$ is an absolutely continuous measure on $[-1,1]$ with weight function

$$
\mu_{b}^{\prime}(x)=\frac{1}{\pi} \frac{1}{\sqrt{1-x^{2}}} \int_{-1}^{1} \frac{\sqrt{1-t^{2}}}{1+x t} d \nu_{0}(t) .
$$

Proof. For orthogonal quadrature the weight function for the orthogonal polynomials is $w_{n}(x)=w(x) e^{-2 n \varphi_{n}(x)}$ with

$$
\varphi_{n}(x)=\frac{1}{2 n}\left[\log \pi_{n+1}(x)+\log \pi_{n}(x)\right],
$$

whereas for Gaussian quadrature it is $w_{n}(x)=w(x) e^{-2 n \psi_{n}(x)}$ with

$$
\psi_{n}=\frac{1}{2 n} \log \pi_{2 n}(x)
$$

By the weak convergence (2.6) we have

$$
\begin{aligned}
\lim _{n \rightarrow \infty} \varphi_{n}(x)= & \lim _{n \rightarrow \infty} \psi_{n}(x)=p \log (1-x)+q \log (1+x) \\
& +[1-(p+q+r)] \int_{-1}^{1} \log (1+t x) d \nu_{0}(t),
\end{aligned}
$$

uniformly on closed sets of $(-1,1)$. By [11, Theorem on p. 124], the asymptotic distribution of the zeros of the corresponding orthogonal polynomials is given by a measure $\mu$ on $[-1,1]$ which is the unique solution of the integral equation

$$
\int_{-1}^{1} \log \frac{1}{|x-t|} d \mu(t)+\varphi(x)=C, \quad x \in[-1,1],
$$

where $\varphi(x)$ is the right-hand side of $(2.10)$ and $C$ is a constant. We easily find

$$
\begin{aligned}
\varphi(x)= & p \log (1-x)+q \log (1+x)-[1-(p+q+r)] \int \log \frac{1}{|x-t|} d \nu_{0}\left(-\frac{1}{t}\right) \\
& +[1-(p+q+r)] \int_{-1}^{1} \log |t| d \nu_{0}(t) .
\end{aligned}
$$

The last term in this expression is a finite constant by (2.7) and may be absorbed by the constant in (2.11). Notice that $\varphi(x)$ may become $-\infty$ near \pm 1 , which at first sight is not allowed when applying the result of [11], but a careful analysis of the proof in [11] shows that the case under consideration is also covered. The integral

$$
\varphi_{b}(x)=\int_{(-\infty,-1) \cup(1, \infty)} \log \frac{1}{|x-t|} d \nu_{0}\left(-\frac{1}{t}\right)
$$

is the logarithmic potential of the mass distribution $\nu_{0}(-1 / t)$ outside $[-1,1]$. The integral equation

$$
\int_{-1}^{1} \log \frac{1}{|x-t|} d \mu_{b}(t)-\varphi_{b}(x)=C, \quad x \in[-1,1]
$$

has a unique solution $\mu_{b}$ which is the 'balayage' of the mass distribution $\nu_{0}\left(-1 / t^{2}\right)$ outside $[-1,1]$ to the interval $[-1,1]$ (see, e.g., $[21$, pp. 126-127]), and this measure $\mu_{b}$ is absolutely continuous with weight function given by (2.9). The arcsin distribution has a constant logarithmic potential on $[-1,1]$, 
which can be absorbed into the constant $C$ in (2.11). Hence, the unique solution of (2.11) is the measure given by $(2.8)$.

Let us take a closer look at the limiting zero distribution measure $\mu$ given by (2.8). If the measure $\nu$ has all its mass at the point $a \in(-1,1)$ then

$$
\mu^{\prime}(x)=\frac{1}{\pi} \frac{1}{\sqrt{1-x^{2}}} \frac{\sqrt{1-a^{2}}}{1+a x} .
$$

If $a=0$, then the limiting zero distribution is the well-known arcsin distribution. For $a=-1$ the limiting zero distribution is a degenerate distribution at 1 , whereas for $a=1$ the limiting zero distribution is concentrated at -1 . If $a$ is close to 1 in (2.12), then $\mu$ has even more mass concentrated around -1 than the arcsin distribution. The measures with weight functions given by (2.9) or (2.12) are thus somewhere between an arcsin measure and degenerate measures at \pm 1 .

Even though the asymptotic distribution of the nodes for orthogonal quadrature and Gaussian quadrature is the same, we see that the $n$ nodes for the Gaussian quadrature formula depend on $2 n$ parameters $t_{i}$, whereas the $n$ nodes for orthogonal quadrature depend on $n+1$ parameters $t_{i}$. The nodes for Gaussian quadrature therefore use more (asymptotic) information of the basis functions.

\section{Properties of the NODES}

The orthogonal functions $r_{n}\left(t_{1}, \ldots, t_{n} ; x\right)(n=1,2, \ldots)$ are an extended Haar system on $[-1,1]$, and therefore the properties of the zeros given by Pinkus, Ziegler, and Videnskii are valid. For this special Haar system, additional useful properties can be proved:

Theorem 3a. (1) The zeros of $r_{n}\left(t_{1}, \ldots, t_{n} ; x\right)$ and $r_{n+1}\left(t_{1}, \ldots, t_{n}, t_{n+1} ; x\right)$ on $[-1,1]$ are strictly interlacing.

(2) The zeros of $r_{n}\left(t_{1}, \ldots, t_{n-1}, t_{n} ; x\right)$ and $r_{n}\left(t_{1}, \ldots, t_{n-1}, t_{n+1} ; x\right)$ on $[-1,1]$ are strictly interlacing.

(3) The zeros of $r_{n}\left(t_{1}, \ldots, t_{i-1}, t_{i+1}, \ldots, t_{n}, t_{i} ; x\right)(1 \leq i \leq n)$ and $r_{n}\left(t_{1}, \ldots, t_{n} ; x\right)$ are strictly interlacing.

Proof. Property (1) is proved in Pinkus and Ziegler [25] and Videnskii [28], and property (2) can be found in Pinkus and Ziegler [25]. For property (3) we notice that

$$
\begin{aligned}
r_{n}\left(t_{1}, \ldots, t_{n} ; x\right)= & r_{n-1}\left(t_{1}, \ldots, t_{i-1}, t_{i+1}, \ldots, t_{n} ; x\right) \\
& -\kappa_{1} r_{n-1}\left(t_{1}, \ldots, t_{i-1}, t_{i+1}, \ldots, t_{n-1}, t_{i} ; x\right)
\end{aligned}
$$

and

$$
\begin{aligned}
& r_{n}\left(t_{1}, \ldots, t_{i-1}, t_{i+1}, \ldots, t_{n}, t_{i} ; x\right) \\
&= r_{n-1}\left(t_{1}, \ldots, t_{i-1}, t_{i+1}, \ldots, t_{n-1}, t_{i} ; x\right) \\
&-\kappa_{2} r_{n-1}\left(t_{1}, \ldots, t_{i-1}, t_{i+1}, \ldots, t_{n-1}, t_{n} ; x\right),
\end{aligned}
$$

where

$$
\kappa_{1}=\frac{\left(r_{n-1}\left(t_{1}, \ldots, t_{i-1}, t_{i+1}, \ldots, t_{n} ; x\right), \frac{1}{1+t_{i} x}\right)}{\left\|r_{n-1}\left(t_{1}, \ldots, t_{i-1}, t_{i+1}, \ldots, t_{n-1}, t_{i} ; x\right)\right\|_{2}^{2}}
$$


and

$$
\kappa_{2}=\frac{\left(r_{n-1}\left(t_{1}, \ldots, t_{i-1}, t_{i+1}, \ldots, t_{n-1}, t_{i} ; x\right), \frac{1}{1+t_{n} x}\right)}{\left\|r_{n-1}\left(t_{1}, \ldots, t_{i-1}, t_{i+1}, \ldots, t_{n} ; x\right)\right\|_{2}^{2}} .
$$

Let $x_{i}(i=1,2, \ldots, n-1)$ be the zeros of $r_{n}\left(t_{1}, \ldots, t_{n} ; x\right)$; then

$$
\begin{aligned}
& r_{n}\left(t_{1}, \ldots, t_{i-1}, t_{i+1}, \ldots, t_{n}, t_{i} ; x_{j}\right) \\
& \quad=r_{n-1}\left(t_{1}, \ldots, t_{i-1}, t_{i+1}, \ldots, t_{n-1}, t_{i} ; x_{j}\right)\left(1-\kappa_{1} \kappa_{2}\right) .
\end{aligned}
$$

If we compute the $L_{2}$-norm of $r_{n}\left(t_{1}, \ldots, t_{n} ; x\right)$, then

$$
\begin{aligned}
\left\|r_{n}\left(t_{1}, \ldots, t_{n} ; x\right)\right\|_{2}^{2}=\left(r_{n}\left(t_{1}, \ldots, t_{n} ; x\right), \frac{1}{1+t_{n} x}\right) \\
=\left(r_{n-1}\left(t_{1}, \ldots, t_{i-1}, t_{i+1}, \ldots, t_{n} ; x\right), \frac{1}{1+t_{n} x}\right) \\
\quad-\kappa_{1}\left(r_{n-1}\left(t_{1}, \ldots, t_{i-1}, t_{i+1}, \ldots, t_{n-1}, t_{i} ; x\right), \frac{1}{1+t_{n} x}\right) \\
=\left\|r_{n-1}\left(t_{1}, \ldots, t_{i-1}, t_{i+1}, \ldots, t_{n} ; x\right)\right\|_{2}^{2}\left(1-\kappa_{1} \kappa_{2}\right)
\end{aligned}
$$

so that $1-\kappa_{1} \kappa_{2}>0$. Then property (3) follows immediately from (3.1) and property (1).

We have similar results for the nodes corresponding to Gaussian quadrature. Now the order of the parameters $t_{1}, \ldots, t_{2 n}$ is irrelevant.

Theorem 3b. Let $x_{i}\left(t_{1}, \ldots, t_{2 n}\right) \quad(i=1,2, \ldots, n)$ be the quadrature nodes for maximum accuracy.

(1) The $n$ nodes $x_{i}\left(t_{1}, \ldots, t_{2 n}\right)$ and the $n+1$ nodes $x_{i}\left(t_{1}, \ldots, t_{2 n+1}, t_{2 n+2}\right)$ are strictly interlacing.

(2) The $n$ nodes $x_{i}\left(t_{1}, \ldots, t_{2 n-1}, t_{2 n}\right)$ and $x_{i}\left(t_{1}, \ldots, t_{2 n-1}, t_{2 n+1}\right)$ are strictly interlacing.

Proof of $(1)$. Let $x_{0}\left(t_{1}, \ldots, t_{2 n}\right)=-1$ and $x_{n+1}\left(t_{1}, \ldots, t_{2 n}\right)=1$.

Suppose that the nodes $x_{i}\left(t_{1}, \ldots, t_{2 n}\right)$ and $x_{i}\left(t_{1}, \ldots, t_{2 n+2}\right)$ are not strictly interlacing; then either

(a) $x_{i}\left(t_{1}, \ldots, t_{2 n}\right)=x_{j}\left(t_{1}, \ldots, t_{2 n+2}\right)$ for some $i$ and $j$ with $1 \leq i \leq n$ and $1 \leq j \leq n+1$, or

(b)

$$
x_{j}\left(t_{1}, \ldots, t_{2 n+2}\right), x_{j+1}\left(t_{1}, \ldots, t_{2 n+2}\right) \in\left(x_{i}\left(t_{1}, \ldots, t_{2 n}\right), x_{i+1}\left(t_{1}, \ldots, t_{2 n}\right)\right)
$$

for some $i$ and $j$ with $0 \leq i \leq n$ and $1 \leq j \leq n$.

We will show that both (a) and (b) are impossible.

Case (a). Suppose that there are $\ell$ nodes $x_{i}\left(t_{1}, \ldots, t_{2 n+2}\right), i \in\left\{i_{1}, i_{2}, \ldots\right.$, $\left.i_{\ell}\right\}$, such that $x_{i}\left(t_{1}, \ldots, t_{2 n+2}\right) \in\left\{x_{j}\left(t_{1}, \ldots, t_{2 n}\right): 1 \leq j \leq n\right\}$; then obviously $\ell \leq n$, and thus there exists a $k$ such that $x_{k}\left(t_{1}, \ldots, t_{2 n+2}\right) \notin$ $\left\{x_{j}\left(t_{1}, \ldots, t_{2 n}\right): 1 \leq j \leq n\right\}$. Let $\phi$ be a linear combination of the first $2 n$ basis functions given in (1.1) such that $\phi\left(x_{i}\left(t_{1}, \ldots, t_{2 n}\right)\right)=0$ for $i=$ $1,2, \ldots, n$ and $\phi\left(x_{i}\left(t_{1}, \ldots, t_{2 n+2}\right)\right)=0$ for $i=1,2, \ldots, 2 n+1$ but $i \neq k$. Then we already have $2 n-\ell$ equations to determine $\phi$. In addition we add 
the equations $\phi\left(z_{i}\right)=0$ for $i=1, \ldots, \ell-1$, where the $z_{i}$ differ from the $2 n-\ell$ points that we already used. For such $\phi$ we have

$$
\int_{-1}^{1} \phi(x) w(x) d x=\sum_{i=1}^{n} \beta_{i}\left(t_{1}, \ldots, t_{2 n}\right) \phi\left(x_{i}\left(t_{1}, \ldots, t_{2 n}\right)\right)=0 .
$$

But we also have

$$
\begin{aligned}
\int_{-1}^{1} \phi(x) w(x) d x & =\sum_{i=1}^{n+1} \beta_{i}\left(t_{1}, \ldots, t_{2 n+2}\right) \phi\left(x_{i}\left(t_{1}, \ldots, t_{2 n+2}\right)\right) \\
& =\beta_{k}\left(t_{1}, \ldots, t_{2 n+2}\right) \phi\left(x_{k}\left(t_{1}, \ldots, t_{2 n+2}\right)\right) \neq 0,
\end{aligned}
$$

because $\phi$ can have at most $2 n-1$ zeros and $\beta_{k}>0$. This gives a contradiction.

Case (b). Let $\phi$ be the linear combination of the first $2 n$ basis functions given in (1.1) such that $\phi\left(x_{i}\left(t_{1}, \ldots, t_{2 n}\right)\right)=0$ for $i=1,2, \ldots, n$, and $\phi\left(x_{i}\left(t_{1}, \ldots, t_{2 n+2}\right)\right)=0$ for $i=1,2, \ldots, n+1$ but with $i \neq j$ and $i \neq j+1$. This gives $2 n-1$ conditions to determine $\phi$, and for such $\phi$ we have

$$
\int_{-1}^{1} \phi(x) w(x) d x=\sum_{i=1}^{n} \beta_{i}\left(t_{1}, \ldots, t_{2 n}\right) \phi\left(x_{i}\left(t_{1}, \ldots, t_{2 n}\right)\right)=0 .
$$

On the other hand, we also have

$$
\begin{aligned}
\int_{-1}^{1} \phi(x) w(x) d x= & \sum_{i=1}^{n+1} \beta_{i}\left(t_{1}, \ldots, t_{2 n+2}\right) \phi\left(x_{i}\left(t_{1}, \ldots, t_{2 n+2}\right)\right) \\
= & \beta_{j}\left(t_{1}, \ldots, t_{2 n+2}\right) \phi\left(x_{j}\left(t_{1}, \ldots, t_{2 n+2}\right)\right) \\
& +\beta_{j+1}\left(t_{1}, \ldots, t_{2 n+2}\right) \phi\left(x_{j+1}\left(t_{1}, \ldots, t_{2 n+2}\right)\right) .
\end{aligned}
$$

By assumption we have

$$
\operatorname{sign} \phi\left(x_{j}\left(t_{1}, \ldots, t_{2 n+2}\right)\right)=\operatorname{sign} \phi\left(x_{j+1}\left(t_{1}, \ldots, t_{2 n+2}\right)\right),
$$

and since the weights $\beta_{j}$ and $\beta_{j+1}$ are positive, we have a contradiction.

Proof of (2). Suppose that

$$
x_{i} \equiv x_{i}\left(t_{1}, \ldots, t_{2 n}\right) \quad \text { and } \quad y_{i} \equiv x_{i}\left(t_{1}, \ldots, t_{2 n-1}, t_{2 n+1}\right)
$$

are not strictly interlacing; then either

(a) $x_{i}=y_{i}$ for all $i=1,2, \ldots, n$; or

(b) $x_{i}=y_{j}$ for some $i$ and $j$ with $1 \leq i \leq n$ and $1 \leq j \leq n$; or

(c) $y_{j}, y_{j+1} \in\left(x_{i}, x_{i+1}\right)$ for some $i$ and $j$ with $0 \leq i \leq n$ and $1 \leq j \leq$ $n-1$.

Case (a). The system of basis functions (1.1) is a Haar system on $[-1,1]$, hence we can find a function $f_{k}$ in the linear space spanned by the first $2 n-1$ basis function in (1.1) such that

$$
\begin{array}{ll}
f_{k}\left(x_{i}\right)=\delta_{i, k}, & i=1,2, \ldots, n, \\
f_{k}\left(\xi_{i}\right)=0, & i=1,2, \ldots, n-1,
\end{array}
$$

where $\xi_{i} \in(-1,1) \backslash\left\{x_{1}, x_{2}, \ldots, x_{n}\right\}$ are $n-1$ arbitrary points. Then, on the one hand,

$$
\int_{-1}^{1} f_{k}(x) w(x) d x=\sum_{i=1}^{n} \beta_{i}\left(t_{1}, \ldots, t_{2 n}\right) f_{k}\left(x_{i}\right)=\beta_{k}\left(t_{1}, \ldots, t_{2 n}\right),
$$


and on the other hand (since $x_{i}=y_{i}$ for $1 \leq i \leq n$ )

$$
\begin{aligned}
\int_{-1}^{1} f_{k}(x) w(x) d x & =\sum_{i=1}^{n} \beta_{i}\left(t_{1}, \ldots, t_{2 n-1}, t_{2 n+1}\right) f_{k}\left(x_{i}\right) \\
& =\beta_{k}\left(t_{1}, \ldots, t_{2 n-1}, t_{2 n+1}\right) .
\end{aligned}
$$

Hence, $\beta_{i}\left(t_{1}, \ldots, t_{2 n-1}, t_{2 n+1}\right)=\beta_{i}\left(t_{1}, \ldots, t_{2 n}\right)$ for $i=1,2, \ldots, n$. Therefore, the quadrature formula will give the exact result for every function $\frac{1}{1+t_{i} x}$ with $i=1,2, \ldots, 2 n+1$. Let $\phi$ be a linear combination of these $2 n+1$ basis functions such that $\phi\left(x_{i}\right)=0$ and $\phi^{\prime}\left(x_{i}\right)=0 \quad(i=1,2, \ldots, n)$; then $\phi$ does not change sign on $[-1,1]$ because $\phi(x)= \pm p_{n}^{2}(x) / \pi_{2 n+1}(x)$ with $p_{n}$ a polynomial of degree $n$ and $\pi_{2 n+1}$ given by (2.2). This implies that

$$
\int_{-1}^{1} \phi(x) w(x) d x \neq 0 \text {. }
$$

This contradicts

$$
\int_{-1}^{1} \phi(x) w(x) d x=\sum_{i=1}^{n} \beta_{i} \phi\left(x_{i}\right)=0 .
$$

Cases (b) and (c). One can obtain a contradiction in a way similar to cases (a) and (b) of (1).

The following result of Markov (see, e.g., [26, Theorem 6.12.1 on p. 115]) allows us to give more information on the behavior of the nodes $x_{i}\left(t_{1}, \ldots, t_{n}, t\right)$ for orthogonal quadrature and $x_{i}\left(t_{1}, \ldots, t_{2 n-1}, t\right)$ for Gaussian quadrature, as functions of $t \in(-1,1)$.

Theorem (Markov). Let $w(x, t)$ be a weight function on $[a, b]$ depending on a parameter $t$ such that $w(x, t)$ is positive and continuous for $a<x<b$ and $c<t<d$. Suppose that $w_{t}(x, t)=\frac{\partial}{\partial t} w(x, t)$ exists and is continuous for $a<x<b$ and $c<t<d$, and that the moments

$$
\int_{a}^{b} x^{k} w_{t}(x, t) d x, \quad k=0,1,2, \ldots, 2 n-1,
$$

converge uniformly in every closed subinterval $c^{\prime} \leq t \leq d^{\prime}$ of $(c, d)$. If the zeros of the orthogonal polynomial $p_{n}(x, t)$ with weight function $w(x, t)$ are denoted by $x_{i}(t) \quad(i=1,2, \ldots, n)$ and if $w_{t}(x, t) / w(x, t)$ is a strictly increasing (decreasing) function of $x \in(a, b)$, then $x_{i}(t)$ is a continuously differentiable and strictly increasing (decreasing) function of $t \in(c, d)$ for every fixed $i$.

Theorem 4. (1) If $x_{i}\left(t_{1}, \ldots, t_{n}, t\right)$ is the ith node for orthogonal quadrature with $n$ nodes, then $x_{i}\left(t_{1}, \ldots, t_{n}, t\right)$ is a continuously differentiable and strictly decreasing function of $t \in(-1,1)$.

(2) Every node $x_{i}\left(t_{1}, \ldots, t_{2 n-1}, t\right)$ for Gaussian quadrature with $n$ nodes is a continuously differentiable and strictly decreasing function of $t \in(-1,1)$.

Proof. By Theorem 1(1) we know that $x_{i}\left(t_{1}, \ldots, t_{n}, t\right)$ is the $i$ th zero of the orthogonal polynomial $p_{n}(x, t)$ of degree $n$ for the weight function

$$
w(x, t)=\frac{w(x)}{\pi_{n}^{2}(x)(1+t x)} .
$$


A simple computation gives

$$
\frac{w_{t}(x, t)}{w(x, t)}=-\frac{x}{1+t x}
$$

and this is a decreasing function of $x \in(-1,1)$. The result thus follows by Markov's theorem.

Similarly, by Theorem 1(2), for the nodes $x_{i}\left(t_{1}, \ldots, t_{2 n-1}, t\right)$ of Gaussian quadrature one can use Markov's theorem for the weight function

$$
w(x, t)=\frac{w(x)}{\pi_{2 n-1}(x)(1+t x)}
$$

to obtain the desired result.

We can also give some monotonicity results for the first and last quadrature coefficients. Let $\beta_{i}\left(t_{1}, \ldots, t_{n}, t\right)$ be the quadrature coefficients corresponding to the nodes $x_{i}\left(t_{1}, \ldots, t_{n}, t\right)$ for orthogonal quadrature with $n$ nodes, and let $t_{1}=0$; then the first quadrature coefficient $\beta_{1}\left(t_{1}, \ldots, t_{n}, t\right)$ is a strictly decreasing continuously differentiable function of $t \in(-1,1)$, and the last quadrature coefficient $\beta_{n}\left(t_{1}, \ldots, t_{n}, t\right)$ is a strictly increasing continuously differentiable function of $t \in(-1,1)$. Similarly, if $\beta_{i}\left(t_{1}, \ldots, t_{2 n-1}, t\right)$ are the quadrature coefficients for Gaussian quadrature with $n$ nodes (with $\left.t_{1}=0\right)$, then $\beta_{1}\left(t_{1}, \ldots, t_{2 n-1}, t\right)$ is a strictly decreasing continuously differentiable function of $t \in(-1,1)$ and $\beta_{n}\left(t_{1}, \ldots, t_{2 n-1}, t\right)$ is a strictly increasing continuously differentiable function of $t \in(-1,1)$. The proofs of these results are rather technical (see [27]) and since only the extreme quadrature weights are considered we do not include them here.

\section{CONVERgence RESUlts}

If the integral $\int_{-1}^{1} f(x) w(x) d x$ is approximated by the orthogonal quadrature sum with $n$ nodes,

$$
\sum_{j=1}^{n} \beta_{i}\left(t_{1}, \ldots, t_{n+1}\right) f\left(x_{i}\left(t_{1}, \ldots, t_{n+1}\right)\right),
$$

we denote the error by

$$
E_{n}^{o} f=\int_{-1}^{1} f(x) w(x) d x-\sum_{j=1}^{n} \beta_{i}\left(t_{1}, \ldots, t_{n+1}\right) f\left(x_{i}\left(t_{1}, \ldots, t_{n+1}\right)\right) .
$$

The error of the Gaussian quadrature formula is denoted by

$$
E_{n}^{g} f=\int_{-1}^{1} f(x) w(x) d x-\sum_{j=1}^{n} \beta_{i}\left(t_{1}, \ldots, t_{2 n}\right) f\left(x_{i}\left(t_{1}, \ldots, t_{2 n}\right)\right) .
$$

It is clear that these quadrature formulas are only going to be relevant if for a large enough class of functions $f$ one has $E_{n}^{o} f \rightarrow 0$ as $n \rightarrow \infty$ and/or $E_{n}^{g} f \rightarrow 0$ as $n \rightarrow \infty$. It is well known that the sequence of basis functions (1.1) is dense in $C[-1,1]$ with respect to the uniform norm if and only if

$$
\sum_{k=1}^{\infty}\left(1-\left|c_{k}\right|\right)=\infty
$$


where $c_{k}=-1 / t_{k}-\sqrt{1 / t_{k}^{2}-1}[1$, p. $254, \S 7] .^{1}$ The following result is straightforward, but we mention it for the sake of completeness.

Theorem 5. Let $t_{1}, t_{2}, t_{3}, \ldots$ be distinct parameters in $(-1,1)$ and $f_{n+1}(x)$ be the best approximation of $f \in C[-1,1]$ using linear combinations of $\frac{1}{1+t_{1} x}$, $\frac{1}{1+t_{2} x}, \ldots, \frac{1}{1+t_{n+1} x}$.

(1) If $t_{1}=0$, then for orthogonal quadrature, there exists a positive constant $M$ not depending on $n$ such that

$$
\left|E_{n}^{o} f\right| \leq M\left\|f-f_{n+1}\right\|_{\infty} .
$$

As a consequence, $E_{n}^{o} f \rightarrow 0$ as $n \rightarrow \infty$ for every bounded Riemann integrable function on $[-1,1]$ whenever (4.1) holds.

(2) For Gaussian quadrature we have similarly

$$
\left|E_{n}^{g} f\right| \leq M\left\|f-f_{2 n}\right\|_{\infty} .
$$

As a consequence, $E_{n}^{g} f \rightarrow 0$ as $n \rightarrow \infty$ for every bounded Riemann integrable function on $[-1,1]$ whenever (4.1) holds.

Proof. The result for orthogonal quadrature can be proved as in the case of polynomial interpolation [8, pp. 126-129]. For Gaussian quadrature we only need to show that $\sum_{i=1}^{n} \beta_{i}\left(t_{1}, \ldots, t_{2 n}\right)$ is bounded. If $t_{1}=0$, then

$$
\sum_{i=1}^{n} \beta_{i}\left(t_{1}, \ldots, t_{2 n}\right)=\int_{-1}^{1} w(x) d x .
$$

If $t_{1} \neq 0$, then

$$
\int_{-1}^{1} \frac{w(x)}{1+t_{1} x} d x=\sum_{i=1}^{n} \frac{\beta_{i}}{1+t_{1} x_{i}}
$$

The function $1 /\left(1+t_{1} x\right)$ has the minimum value $1 /\left(1+\left|t_{1}\right|\right)$ on $[-1,1]$, hence

$$
\frac{1}{1+\left|t_{1}\right|} \sum_{i=1}^{n} \beta_{i}\left(t_{1}, \ldots, t_{2 n}\right)<\int_{-1}^{1} \frac{w(x)}{1+t_{1} x} d x
$$

which shows the desired boundedness.

Let $f \in C[-1,1]$ and denote by $L_{n} f$ the rational interpolant of $f$ at the points $x_{i}\left(t_{1}, \ldots, t_{n+1}\right) \quad(i=1,2, \ldots, n)$ using the basis functions $\frac{1}{1+t_{1} x}$, $\frac{1}{1+t_{2} x}, \ldots, \frac{1}{1+t_{n} x}$. The previous theorem implies that

$$
\lim _{n \rightarrow \infty} \int_{-1}^{1}\left(L_{n} f\right)(x) w(x) d x=\int_{-1}^{1} f(x) w(x) d x,
$$

whenever (4.1) holds. The rate of convergence depends on how well $f$ can be approximated in the uniform norm by the basis functions (1.1), and this depends on the choice of the parameters $t_{i}$. We now show that rational interpolation converges in $L_{2}(w)$ and thus also in $L_{1}(w)$, thereby extending the famous Erdős-Turán result for polynomial interpolation [6].

${ }^{1}$ There is a misprint in this reference: 'interior' should be replaced by 'exterior'. We thank P. Borwein for pointing out this misprint. 
Theorem 6. Suppose $t_{1}=0$ and $t_{2}, t_{3}, \ldots$ are distinct parameters in $(-1,1)$ such that (4.1) holds. If $f \in C[-1,1]$ and if $L_{n} f(x)=\sum_{i=1}^{n} \frac{a_{i}}{1+t_{i} x}$ interpolates $f$ at the zeros $x_{i}\left(t_{1}, \ldots, t_{n+1}\right)$ of $r_{n+1}\left(t_{1}, \ldots, t_{n+1} ; x\right)$, then $\left\|L_{n} f-f\right\|_{2} \rightarrow 0$ as $n \rightarrow \infty$.

Proof. Let $f_{n}(x)$ be the best uniform approximation of $f$ using the basis functions $\left\{\frac{1}{1+t_{1} x}, \ldots, \frac{1}{1+t_{n} x}\right\}$; then

$$
\left\|L_{n} f-f\right\|_{2} \leq\left\|f-f_{n}\right\|_{2}+\left\|L_{n} f-f_{n}\right\|_{2} .
$$

Clearly, $\left\|f-f_{n}\right\|_{2} \rightarrow 0$ as $n \rightarrow \infty$. Now $\left[\left(L_{n} f\right)(x)-f_{n}(x)\right]^{2}$ is a linear combination of the functions $x^{j} /\left[\pi_{n}^{2}(x)\right](j=0,1, \ldots, 2 n-2)$, and by $(2.4)$ the quadrature formula is therefore exact for $\left[\left(L_{n} f\right)(x)-f_{n}(x)\right]^{2}$. This gives

$$
\begin{aligned}
\left\|L_{n} f-f_{n}\right\|_{2}^{2} & =\sum_{i=1}^{n} \beta_{i}\left[\left(L_{n} f\right)\left(x_{i}\right)-f_{n}\left(x_{i}\right)\right]^{2} \\
& =\sum_{i=1}^{n} \beta_{i}\left[f\left(x_{i}\right)-f_{n}\left(x_{i}\right)\right]^{2} \leq\left\|f-f_{n}\right\|_{\infty}^{2} \int_{-1}^{1} w(x) d x .
\end{aligned}
$$

The result now follows because $\left\|f-f_{n}\right\|_{\infty} \rightarrow 0$ as $n \rightarrow \infty$.

\section{Generalizations}

All the properties and results for orthogonal quadrature that we have given above are also true for the orthogonal system obtained by orthogonalizing the system of basis functions

$$
\frac{1}{1+t_{i} x},\left.\frac{\partial}{\partial t} \frac{1}{1+t x}\right|_{t=t_{i}}, \ldots,\left.\frac{\partial^{\mu_{i}-1}}{\partial t^{\mu_{i}-1}} \frac{1}{1+t x}\right|_{t=t_{i}}, \quad i=1, \ldots, k,
$$

where $k \in \mathbb{N}, \mu_{i} \in \mathbb{N}$, and $\sum_{i=1}^{k} \mu_{i}=n+1$. If the parameters $t_{i}$ are all distinct and in $(-1,1)$, then this system of functions is an extended Haar system on $[-1,1]$. If $t \notin\left\{t_{1}, \ldots, t_{k}\right\}$, then

$$
r_{n+1}\left(t_{1}^{\left(\mu_{1}\right)}, \ldots, t_{k}^{\left(\mu_{k}\right)}, t ; x\right)=\frac{1}{1+t x}+\left.\sum_{i=1}^{k} \sum_{j=0}^{\mu_{i}-1} a_{i, j} \frac{\partial^{j}}{\partial t^{j}} \frac{1}{1+t x}\right|_{t=t_{i}}
$$

is orthogonal to the system (5.1) with respect to the weight function $w(x)$; if $t=t_{\ell}$ for some $\ell$ with $1 \leq \ell \leq k$, then

$$
\begin{aligned}
& r_{n+1}\left(t_{1}^{\left(\mu_{1}\right)}, \ldots, t_{\ell-1}^{\left(\mu_{\ell-1}\right)}, t_{\ell+1}^{\left(\mu_{\ell+1}\right)}, \ldots, t_{k}^{\left(\mu_{k}\right)}, t_{\ell}^{\left(\mu_{\ell}+1\right)} ; x\right) \\
& \quad=\left.\frac{\partial^{\mu_{\ell}}}{\partial t^{\mu_{\ell}}} \frac{1}{1+t x}\right|_{t=t_{\ell}}+\left.\sum_{i=1}^{k} \sum_{j=0}^{\mu_{i}-1} a_{i, j} \frac{\partial^{j}}{\partial t^{j}} \frac{1}{1+t x}\right|_{t=t_{i}}
\end{aligned}
$$

is orthogonal to the system (5.1) with respect to the weight function $w(x)$. Because of the Haar property it follows that these orthogonal functions $r_{n+1}$ have precisely $n$ zeros in $[-1,1]$ which are all simple $[25,28]$. These orthogonal functions share the same properties as the orthogonal functions for the system 
(1.1): the reason for this is that Theorem 1 can be extended to this system. Similarly, all the results and properties for Gaussian quadrature that we have given are also true for the system of basis functions (5.1) with $k \in \mathbb{N}, \mu_{i} \in \mathbb{N}$, and $\sum_{i=1}^{k} \mu_{i}=2 n$. The only things that change are the weight functions (2.1) and $(2.3)$, which need to be appropriately modified.

Theorem 7. (1) The zeros of $r_{n+1}\left(t_{1}^{\left(\mu_{1}\right)}, \ldots, t_{k}^{\left(\mu_{k}\right)} ; x\right)$ are also the zeros of the orthogonal polynomial $p_{n}(x)$ of degree $n$ for the weight function

$$
\frac{w(x)}{\prod_{i=1}^{k-1}\left(1+t_{i} x\right)^{2 \mu_{i}}\left(1+t_{k} x\right)^{2 \mu_{k}-1}} \text {. }
$$

(2) The $n$ nodes for Gaussian quadrature with the system (5.1) are the zeros of the orthogonal polynomial $p_{n}(x)$ of degree $n$ for the weight function

$$
\frac{w(x)}{\prod_{i=1}^{k}\left(1+t_{i} x\right)^{\mu_{i}}} .
$$

This theorem allows us to use Markov's theorem to deduce properties of the nodes and the weights for orthogonal quadrature, but since everything is along the same lines as before, we do not give the details.

\section{NUMERICAL EXAMPLES}

From Theorem 5 it follows that rational quadrature rules will be effective whenever the function to be integrated can be approximated well using the basis functions (1.1). Gautschi [9] has shown that functions which have an infinite number of poles but are regular otherwise, can be integrated most effectively by Gauss rational quadrature rules by choosing the parameters $t_{i}$ in an optimal way. Gautschi [10] explicitly used such quadrature rules for the computation of generalized Fermi-Dirac and Bose-Einstein integrals.

For functions with most mass concentrated near the endpoints of the interval $[-1,1]$, one could choose the parameters $t_{i}$ in such a way that more quadrature nodes are situated near \pm 1 . If we take the parameters

$$
t_{i}=1-\frac{1}{\sqrt{i}}, \quad i \in \mathbb{N}
$$

then there will be more quadrature nodes near -1 than near +1 . In fact, from Theorem 2 it follows that the asymptotic distribution of the nodes for both orthogonal quadrature and Gaussian quadrature is degenerate at the point -1 . Let $\operatorname{GR}(n)$ be the $n$-point Gaussian quadrature rule, and $\mathrm{OR}(n)$ the $n$-point orthogonal quadrature rule. The nodes and weights for $n=6$ are given in Table 1. Our computations have been made on an IBM 3090/400E VF using Brent's multiple precision package [5], and an independent computation was made using Mathematica ${ }^{\mathrm{TM}}$ [29]. ${ }^{2}$ The nodes and weights for $\mathrm{GR}(n)$ and

\footnotetext{
${ }^{2}$ MATHEMATica is a trademark of Wolfram Research, Inc.
} 
TABLE 1. Nodes and weights for the quadrature rules GR(6) and OR(6)

\begin{tabular}{|c|c|}
\hline nodes for GR $(6)$ & weights for GR $(6)$ \\
\hline-0.9797390942708352 & 0.0528758827013522 \\
-0.8853794251591486 & 0.1411615118844550 \\
-0.6822351336410264 & 0.2748067575758422 \\
-0.3156675377072605 & 0.4657849717765712 \\
0.2408527285476740 & 0.6221630733806293 \\
0.8155273184304977 & 0.4432078026811501 \\
\hline \hline nodes for OR $(6)$ & weights for OR $(6)$ \\
\hline-0.9736320979338328 & 0.0685126325838336 \\
-0.8537169072027923 & 0.1760476819554412 \\
-0.6094091127142633 & 0.3192517203251832 \\
-0.2057016948376719 & 0.4878639628808742 \\
0.3414560761423378 & 0.5765658940369015 \\
0.8474273771128526 & 0.3717581082177663 \\
\hline
\end{tabular}

$\mathrm{OR}(n)$ have been computed by using the zeros and Christoffel numbers for the orthogonal polynomial of degree $n$ with the corresponding weight (2.1) for orthogonal quadrature and (2.3) for Gaussian quadrature. These orthogonal polynomials have been computed using their three-term recurrence relation. The recurrence coefficients were obtained using Chebyshev's algorithm with modified moments; the latter were computed recursively by decomposing the rational weight function into simple fractions.

Consider the integral

$$
I_{1}=\int_{-1}^{1} \omega e^{-\omega(x+1)} d x, \quad \omega>0 .
$$

This function has more mass near -1 than near +1 , especially for large $\omega$. In Table 2 we have given the absolute values of the relative errors for the Gaussian quadrature sum and the orthogonal quadrature sum corresponding to the rational functions with parameters given by (6.1), together with the Gauss-Legendre quadrature sum $\mathrm{GL}(n)$ with $n$ nodes. The rational quadrature rules behave similarly and give better results than the Gauss-Legendre quadrature rule.

TABLE 2. Relative errors for $I_{1}$

\begin{tabular}{|ll|lll|}
\hline$\omega$ & $n$ & $\mathrm{GR}(n)$ & $\mathrm{OR}(n)$ & $\mathrm{GL}(n)$ \\
\hline 5 & 2 & $0.664(-1)$ & 0.121 & 0.394 \\
& 6 & $0.261(-5)$ & $0.207(-6)$ & $0.161(-4)$ \\
& 10 & $0.215(-8)$ & $0.842(-10)$ & $0.212(-5)$ \\
& 14 & $0.494(-11)$ & $0.514(-13)$ & $0.112(-5)$ \\
\hline 25 & 2 & 0.978 & 0.988 & 0.999 \\
& 6 & $0.118(-4)$ & $0.255(-4)$ & 0.206 \\
& 10 & $0.397(-8)$ & $0.467(-8)$ & $0.201(-2)$ \\
& 14 & $0.206(-12)$ & $0.122(-12)$ & $0.153(-4)$ \\
\hline
\end{tabular}


TABLE 3. Relative errors for $I_{2}$

\begin{tabular}{|l|lll|}
\hline$n$ & $\mathrm{GR}(n)$ & $\mathrm{OR}(n)$ & $\mathrm{GL}(n)$ \\
\hline 2 & $0.587(-6)$ & $0.168(-4)$ & $0.366(-2)$ \\
6 & $0.161(-19)$ & $0.262(-16)$ & $0.567(-7)$ \\
10 & $0.986(-31)$ & $0.132(-29)$ & $0.116(-11)$ \\
\hline
\end{tabular}

The rational quadrature rules perform well because the system of rational functions with parameters (6.1) emphasizes the behavior of the integrand near -1 , and even though the quadrature nodes have a degenerate asymptotic distribution at -1 , the parameters still satisfy (4.1), so that the basis functions are dense in $C[-1,1]$. The fact that the poles of the rational functions are all close to -1 also explains why both rational quadrature rules give similar results: adding extra rational functions is not giving much extra information about the integrand.

As a second example we consider the integral

$$
I_{2}=\int_{-1}^{1} \frac{d x}{\sqrt{(x+3)(x+2)}} .
$$

The integrand has a branch cut on the interval $[-3,-2]$ and is in fact a Stieltjes function given by

$$
\frac{1}{\sqrt{(x+3)(x+2)}}=\frac{1}{\pi} \int_{-3}^{-2} \frac{1}{x-t} \frac{d t}{\sqrt{(3+t)(-2-t)}} .
$$

Stieltjes functions can be well approximated by rational functions with poles on the cut. Therefore we will choose the parameters $t_{i}$ in such a way that the poles of the basis functions (1.1) are distributed nicely on [-3, -2]. In order to do this we observe that the zeros of a Chebyshev polynomial of the first kind $T_{3^{k}}(x)$ are also zeros of Chebyshev polynomials $T_{3^{m}}(x)$ for every $m \geq k$. Hence, we take $t_{1}=0$ (so that Theorem 1 can be applied) and $t_{2}, t_{3}, \ldots, t_{3^{m}+1}$ equal to

$$
t_{i+1}=\frac{-2}{x_{i, 3^{m}}-5}
$$

where the $x_{i, 3^{m}}$ are the zeros of the Chebyshev polynomial $T_{3^{m}}(x)$ ordered in such a way that $x_{1,3^{m}}, x_{2,3^{m}}, x_{3,3^{m}}$ are the zeros of $T_{3}(x)$ in decreasing order; $x_{4,3^{m}}, \ldots, x_{9,3^{m}}$ are those zeros of $T_{9}(x)$ which are not zeros of $T_{3}(x)$, again in decreasing order; and recursively we let $x_{3^{k-1}+1,3^{m}}, \ldots, x_{3^{k}, 3^{m}}$ be the zeros of $T_{3^{k}}(x)$ which are not zeros of $T_{3^{k-1}}(x)$, in decreasing order. In this way the poles of the basis functions $(1.1)$ are dense on $[-3,-2]$ and they are distributed on $[-3,-2]$ according to the arcsin measure on this interval. In Table 3 we have given the relative errors for the two rational quadrature rules and for the Gauss-Legendre rule.

It is clear that the rational quadrature rules give a much better approximation to the integral $I_{2}$ than the Gauss-Legendre rule. The reason is obvious: the integrand is a Stieltjes function which can be approximated much better by rational functions than by polynomials, especially when we choose the poles of the rational functions on the interval $[-3,-2]$ on which the Stieltjes function 
TABLE 4. Relative errors for $I_{3}$

\begin{tabular}{|ll|lll|}
\hline$\omega$ & $n$ & $\mathrm{GR}(n)$ & $\mathrm{OR}(n)$ & $\mathrm{GL}(n)$ \\
\hline 2 & 5 & $0.46(-11)$ & $0.26(-8)$ & $0.52(-5)$ \\
& 10 & $0.16(-24)$ & $0.79(-22)$ & $0.10(-10)$ \\
& 15 & - & $0.17(-24)$ & $0.20(-16)$ \\
\hline 1.1 & 5 & $0.21(-8)$ & $0.26(-5)$ & $0.21(-1)$ \\
& 10 & $0.19(-22)$ & $0.30(-16)$ & $0.26(-3)$ \\
& 15 & $0.81(-25)$ & $0.67(-25)$ & $0.32(-5)$ \\
\hline 1.01 & 5 & $0.43(-8)$ & $0.93(-5)$ & 0.24 \\
& 10 & $0.91(-22)$ & $0.24(-15)$ & $0.64(-1)$ \\
& 15 & $0.40(-26)$ & $0.61(-26)$ & $0.17(-1)$ \\
\hline
\end{tabular}

TABLE 5. Relative errors for $I_{4}$

\begin{tabular}{|ll|lll|}
\hline$\omega$ & $n$ & $\mathrm{GR}(n)$ & $\mathrm{OR}(n)$ & $\mathrm{GL}(n)$ \\
\hline 2 & 5 & $0.65(-5)$ & $0.79(-8)$ & $0.47(-4)$ \\
& 10 & $0.12(-10)$ & $0.24(-21)$ & $0.19(-9)$ \\
& 15 & $0.22(-16)$ & $0.15(-24)$ & $0.56(-15)$ \\
\hline 1.1 & 5 & $0.17(-1)$ & $0.27(-5)$ & 0.13 \\
& 10 & $0.32(-3)$ & $0.30(-16)$ & $0.34(-2)$ \\
& 15 & $0.38(-5)$ & $0.27(-24)$ & $0.62(-4)$ \\
\hline 1.01 & 5 & 0.37 & $0.16(-5)$ & 0.78 \\
& 10 & $0.93(-1)$ & $0.40(-16)$ & 0.40 \\
& 15 & $0.23(-1)$ & $0.11(-24)$ & 0.16 \\
\hline
\end{tabular}

has its singularities. Also the Gaussian quadrature rule is slightly better than the orthogonal rule, because the Gaussian rule with $n$ nodes already uses $2 n-1$ poles on the interval $[-3,-2]$, whereas the orthogonal rule only uses $n$ poles.

Finally we consider the integrals

$$
I_{3}=\int_{-1}^{1} \frac{\pi t / \omega}{\sin (\pi t / \omega)} d t, \quad I_{4}=\int_{-1}^{1}\left(\frac{\pi t / \omega}{\sin (\pi t / \omega)}\right)^{2} d t
$$

Walter Gautschi provided us with numerical results for the approximation of these two integrals with rational quadrature rules. The integrands contain infinitely many poles at the points $p_{1}=\omega, p_{2}=-\omega, p_{3}=2 \omega, p_{4}=-2 \omega, \ldots$. For $I_{3}$ these are simple poles but for $I_{4}$ double poles. For the orthogonal quadrature rule we choose the parameters $t_{i}$ in such a way that they match up with the poles of the integrand, i.e.,

$$
t_{1}=0, \quad t_{i+1}=-1 / p_{i}, \quad i=1,2, \ldots .
$$

For the Gaussian rule we choose $t_{i}=-1 / p_{i}$. The results for Gaussian rational quadrature $\mathrm{GR}(n)$, orthogonal rational quadrature $\mathrm{OR}(n)$, and GaussLegendre quadrature $\operatorname{GL}(n)$ are given in Table 4 and Table 5. ${ }^{3}$ Both rational quadrature rules perform very well for $I_{3}$, which is simple to understand since

\footnotetext{
${ }^{3}$ These computations were done by W. Gautschi on the Cyber 205, with a requested error tolerance of $0.5 \times 10^{-25}$.
} 
these rules explicitly take into account the poles of the integrand. The GaussLegendre rule does not take these singularities into account, and as a result it behaves poorly.

When the integrand has poles of second order, then there is also a difference in the performance of the rational quadrature rules.

For the integral $I_{4}$ the orthogonal quadrature rule $\mathrm{OR}(n)$ works very well, even for $\omega$ close to one. Both the Gauss-Legendre rule GL( $n)$ and the rational Gaussian rule $\operatorname{GR}(n)$ have difficulties when $\omega$ approaches 1 , and even for $\omega=2$ they do not converge as fast as the orthogonal quadrature rule $\operatorname{OR}(n)$. For GL $(n)$ the reason is clear: no poles of the integrand are being taken into account. On the other hand, $\operatorname{GR}(n)$ approximates the integrand by a rational function with simple poles, whereas the integrand actually has double poles. Underestimating the order of the poles thus seems to degrade the convergence. By choosing $q_{2 n-1}(x)=\pi_{n}(x) \pi_{n+1}(x) /\left(1+t_{k} x\right)^{2}$ in (2.4) we see that the orthogonal quadrature rule integrates $\left(1+t_{k} x\right)^{-2}(k=2,3, \ldots, n)$ exactly. Hence the orthogonal rule is more natural when the integrand has poles of order two, but in this case one could also use the Gaussian quadrature rule corresponding to the basis function

$$
\frac{1}{1+t_{1} x}, \frac{1}{\left(1+t_{1} x\right)^{2}}, \frac{1}{1+t_{2} x}, \frac{1}{\left(1+t_{2} x\right)^{2}}, \ldots, \frac{1}{1+t_{n} x}, \frac{1}{\left(1+t_{n} x\right)^{2}},
$$

which by Theorem 7 uses the zeros of the orthogonal polynomials of degree $n$ with weight function $\prod_{i=1}^{n}\left(1+t_{i} x\right)^{-2}$.

\section{ACKNOWLEDGMENT}

We are very grateful to Walter Gautschi for his useful comments on an earlier version of the manuscript and for encouraging us to work out numerical examples. We are especially grateful for the numerical experiments he made in Tables 4 and 5 and for his comments on these results.

\section{BIBLIOGRAPHY}

1. N. I. Achieser, Theory of approximation, Ungar, New York, 1956.

2. D. L. Barrow, On multiple node Gaussian quadrature formulae, Math. Comp. 32 (1979), 431-439.

3. B. D. Bojanov, D. Braess, and N. Dyn, Generalized Gaussian quadrature formulas, J. Approx. Theory 48 (1986), 335-353.

4. D. Braess, Nonlinear approximation theory, Springer-Verlag, Berlin, 1986.

5. R. P. Brent, A FORTRAN multiple-precision arithmetic package, ACM Trans. Math. Software 4 (1978), 57-70.

6. E. W. Cheney, Introduction to approximation theory. II, McGraw-Hill, New York, 1966.

7. P. J. Davis, Interpolation and approximation, Blaisdell, New York, 1965.

8. P. J. Davis and P. Rabinowitz, Methods of numerical integration, 2nd ed., Academic Press, New York, 1984.

9. W. Gautschi, Gauss-type quadrature rules for rational functions, Numerical Integration IV (H. Brass and G. Hämmerlin, eds.) (to appear).

10. Phys. Comm. 74 (1993), 233-238. 
11. A. A. Gonchar and E. A. Rakhmanov, Equilibrium measure and the distribution of zeros of extremal polynomials, Mat. Sb. 125 (167) (1984), 117-127; English transl. in Math. USSR-Sb. 53 (1986), 119-130.

12. S. Karlin and A. Pinkus, Gaussian quadrature formulae with multiple nodes, Studies in Spline Functions and Approximation Theory, Academic Press, New York, 1976, pp. 113142.

13. _ An extremal property of multiple Gaussian nodes, Studies in Spline Functions and Approximation Theory, Academic Press, New York, 1976, pp. 143-162.

14. S. Karlin and W. J. Studden, Tchebycheff systems with applications in analysis and statistics, Interscience, New York, 1966.

15. M. Krein, The ideas of P.L. Chebysheff and A.A. Markov in the theory of limiting values of integrals and their further developments, Uspekhi Fiz. Nauk (1951), 3-120; English transl., Amer. Math. Soc. Transl. (2) 12 (1951), 1-122.

16. G. López Lagomasino, Relative asymptotics for polynomials orthogonal on the real axis, Mat. Sb. 137 (179) (1988); English transl. in Math. USSR-Sb. 65 (1990), 505-529.

17. __ Asymptotics of polynomials orthogonal with respect to varying measures, Constr. Approx. 5 (1989), 199-219.

18. G. López Lagomasino and J. Illán, A note on generalized quadrature formulas of Gauss-Jacobi type, Constructive Theory of Function '84, Sofia, pp. 513-518.

19. __ Sobre los métodos interpolatorios de integración numérica y su conexión con la aproximación racional, Rev. Cienc. Mat. (2) 8 (1987), 31-44.

20. G. López, Szegö's theorem for polynomials orthogonal with respect to varying measures, Orthogonal Polynomials and their Applications, Lecture Notes in Math., vol. 1329, SpringerVerlag, Berlin, 1988, pp. 255-260.

21. G. López and E. A. Rakhmanov, Rational approximations, orthogonal polynomials and equilibrium distributions, Orthogonal Polynomials and their Applications, Lecture Notes in Math., vol. 1329, Springer-Verlag, Berlin, 1988, pp. 125-157.

22. H. N. Mhaskar and E. B. Saff, Weighted polynomials on finite and infinite intervals: a unified approach, Bull. Amer. Math. Soc. (N.S.) 11 (1984), 351-354.

23. __ Where does the sup norm of a weighted polynomial live? (a generalization of incomplete polynomials), Constr. Approx. 1 (1985), 71-91.

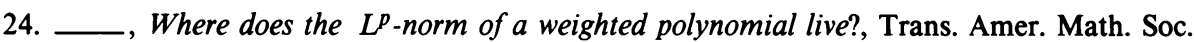
303 (1987), 109-124.

25. A. Pinkus and Z. Ziegler, Interlacing properties of the zeros of the error functions in best $L^{p}$-approximations, J. Approx. Theory 27 (1979), 1-18.

26. G. Szegö, Orthogonal polynomials, 4th ed., Amer. Math. Soc. Colloq. Publ., vol. 23, Amer. Math. Soc., Providence, RI, 1975.

27. H. Van de Vel and I. Vanherwegen, Quadrature formulas based on interpolation using $\gamma$-polynomials, manuscript.

28. V.S. Videnskiī, On zeros of orthogonal polynomials, Soviet Math. Dokl. 4 (1963), 14791482.

29. S. Wolfram, Mathematica ${ }^{T M}$, A system for doing mathematics by computer, AddisonWesley, New York, 1988.

Department of Mathematics, Katholieke Universiteit Leuven, CelestiJnenlaAn 200 B, B-3001 HeVERLEe, BELGIUM

E-mail address: fgaee03@cc1.KULeuven.ac.be 